\title{
Cavitación peripapilar coroidea en el diagnóstico diferencial de lesión pigmentada yuxtapapilar
}

\section{Pigmented juxtapapillary lesion as a form of presentation of parapapillary choroidal cavitation}

\author{
Fernando López-Herrero*, José L. Sánchez-Vicente y Jorge L. Monge-Esquivel
}

Servicio de Oftalmología, Hospital Universitario Virgen del Rocío, Sevilla, España

\section{Resumen}

Las lesiones pigmentadas yuxtapapilares representan un importante reto diagnóstico en oftalmología. Presentamos un caso de lesión pigmentada yuxtapapilar cuyo estudio de imagen multimodal evidenció tratarse de una forma de presentación de una cavitación coroidea peripapilar.

Palabras clave: Cavitación peripapilar coroidea. OCT En-face. OCT Swept Source. Miopía magna.

\begin{abstract}
Juxtapapillary pigmented lesions represent an important diagnostic challenge in ophthalmology. We present a case of a juxtapapillary pigmented lesion whose multimodal imaging study evidenced a form of presentation of a peripapillary choroidal cavitation.
\end{abstract}

Key words: Peripapillary choroid cavitation. En-face OCT. Swept Source OCT. Degenerative myopia.

Fecha de recepción: 17-03-2018

Fecha de aceptación: 04-06-2018 41011, Sevilla, España DOI: 10.24875/RMO.M18000040
Disponible en internet: 01-05-2019 Rev Mex Oftalmol. 2019;93(3):157-158 www.rmo.com.mx 

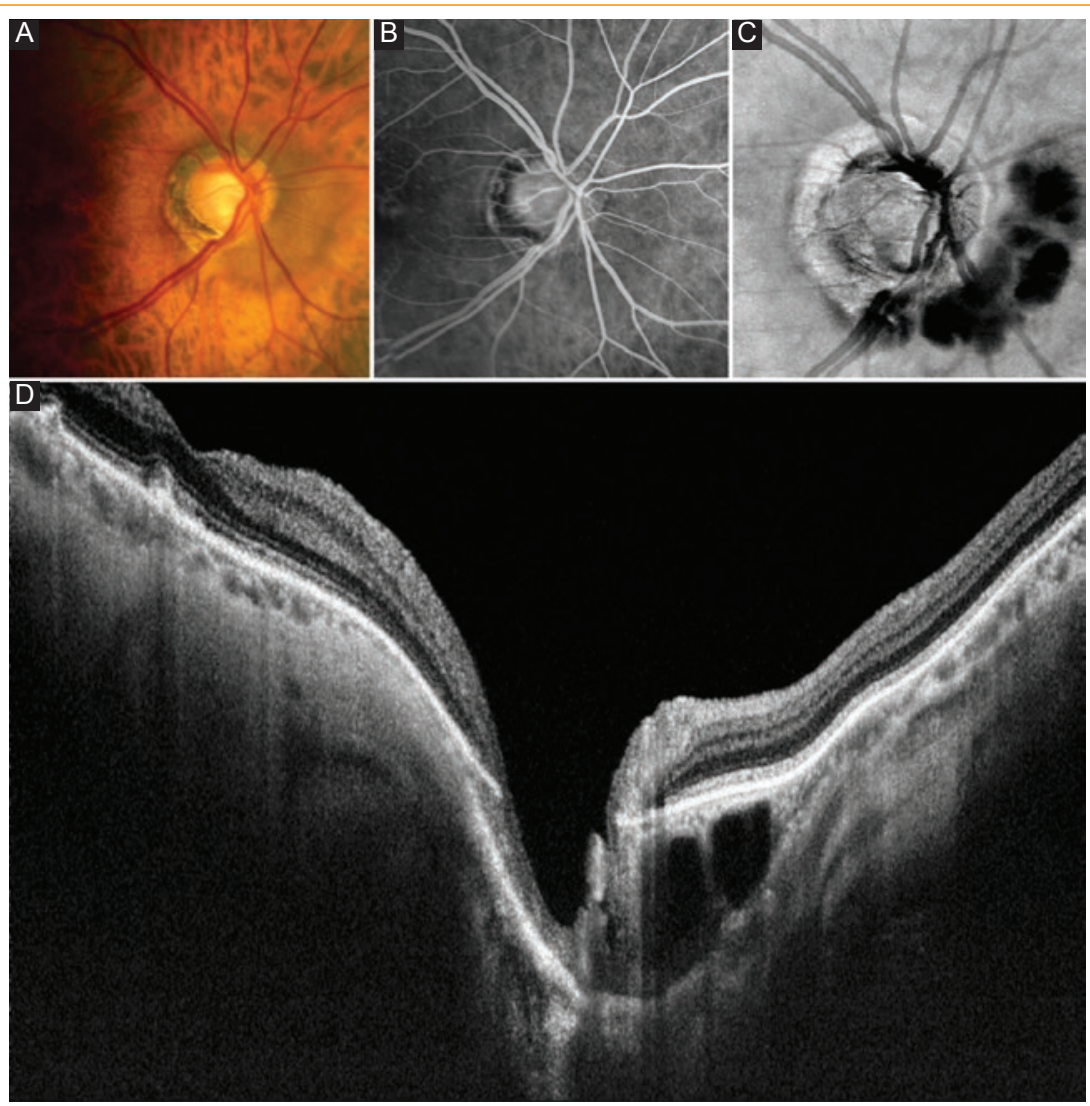

Figure 1. Análisis multimodal de la lesión yuxtapapilar pigmentada. A: Imagen retinográfica; B: Imagen de angiofluoresceingrafía en tiempos precoces; C: Estudio de tomografía de coherencia óptica (OCT) En-face segmentada a nivel coroideo; D: OCT Swept-Source (DRI OCT Triton plus, Topcon Medical Systems, Inc, Europe) con hallazgos característicos de cavitaciones peripapilares coroideas (CPC).

Paciente de 64 años miope de -7 dioptrías. La retinografía del ojo derecho (OD) mostró una lesión pigmentada yuxtapapilar nasal no sobreelevada y bien delimitada (Fig. 1 A). En tiempos precoces de la angiofluoresceingrafía se observó un tenue efecto pantalla (Fig. 1 B). La tomografía de coherencia óptica (OCT) En-face, segmentada a nivel coroideo (Fig.1 C), destacó la presencia de espacios hiporreflectivos, aparentemente compartimentados por septos interpuestos. El estudio OCT Swept-Source (Fig. $1 \mathrm{D}$ ) reveló la imagen característica de las cavitaciones peripapilares coroideas (CPC), en este caso con estafiloma tipo 3 de Curtin'.

En conclusión, frente a la descripción característica anaranjada-amarillenta, las CPC deben también considerarse en el diagnóstico diferencial de las lesiones pigmentadas peripapilares ${ }^{2}$.

\section{Responsabilidades éticas}

Protección de personas y animales. Los autores declaran que para esta investigación no se han realizado experimentos en seres humanos ni en animales.

Confidencialidad de los datos. Los autores declaran que han seguido los protocolos de su centro de trabajo sobre la publicación de datos de pacientes.

\section{Derecho a la privacidad y consentimiento informa-} do. Los autores declaran que en este artículo no aparecen datos de pacientes.

\section{Conflicto de intereses}

Los autores declaran no tener ningún conflicto de intereses.

\section{Bibliografía}

1. Shinohara K, Moriyama M, Shimada N, Yoshida T, Ohno-Matsui K. Characteristics of Peripapillary Staphylomas Associated With High Myopia Determined by Swept-Source Optical Coherence Tomography. Am J Ophthalmol. 2016;169:138-44. doi: 10.1016/j.ajo.2016.06.033. Epub 2016 Jun 27.

2. Yeh SI, Chang WC, Wu CH, Lan YW, Hsieh JW, Tsai S, Chen LJ. Characteristics of peripapillary choroidal cavitation detected by optical coherence tomography. Ophthalmology. 2013;120(3):544-52. 